\title{
A Comparative Study on Cloud Platform Model of Chinese and American Maker Space
}

\author{
Lv Bo, Gu Qiaoling, Zhi Yechao \\ Business School, Beijing Wuzi University, Beijing, China \\ Email address: \\ 1vbo73@sina.com (Lv Bo), gupanpan0131@sina.com (Gu Qiaoling),zyc201408@sina.com (ZhiYechao)
}

\section{To cite this article:}

Lv Bo, Gu Qiaoling, Zhi Yechao. A Comparative Study on Cloud Platform Model of Chinese and American Maker Space. International Journal of Economics, Finance and Management Sciences. Vol. 7, No. 6, 2019, pp. 187-196. doi: 10.11648/j.ijefm.20190706.12

Received: October 11, 2019; Accepted: November 3, 2019; Published: November 8, 2019

\begin{abstract}
This paper constructs a comparison framework of the cloud-based platform model consisting of cloud foundation, cloud function, cloud resource, cloud collaboration and cloud sustainability, and randomly selects 610 of Chinese maker space and American maker space as comparable samples. A comparative study was made on the status quo of its development. The study found that the similarity lies in the fact that both China and the US have rapidly grown in number, creating more than 30 types of cloud functions, gathering more than 80 types of cloud resources, forming a cloud collaboration network, and initially generating a sustainable cloud platform ecosystem. The difference is that the US created a space early, on the cloud to form an advanced infrastructure with 3D printing as the standard, highlighting online interaction on cloud functions, vertical areas on cloud resources, and cloud collaboration pursue intelligent matching and comprehensively utilize up to 12 revenue channels in cloud sustainability. Aiming at the problems existing in Chinese maker space cloud platform model, this paper reveals the external manifestation of the US maker space cloud platform model. It gives enlightenment to China from cloud foundation, cloud function, cloud resource, cloud collaboration and cloud sustainability. And revealing the logic of internal development, and proposing that Chinese mass maker space should be "table" and "inside" and to promote the soundness and development of the model of space cloud platform in China.
\end{abstract}

Keywords: Maker Space, Cloud Platform, Mode, Comparative Study

\section{Introduction}

Since the beginning of 2015, maker space has been officially proposed in the national documents and has been listed as a strong support for development. Since then, Chinese maker space has experienced a spurt of growth. According to the statistics of the Ministry of Science and Technology, by 2018, the number of space created by China has reached more than 5,500. There are five types of formats in the US that are similar to the space created by China. They are Maker Space, Hackerspace, WeWork, TechShop, and Fabric Lab. [1, 2] According to the research in this paper, the US maker space has been mushrooming in the past three years, and its number has exceeded 500. Together with the other four types of space creation, the total number of maker spaces in the US exceeds 1,200. Although the maker space in the US does not predominate in quantity, it starts earlier than China, and its external manifestation and internal development logic are worth learning. The maker space cloud platform model has attracted the attention of China and the US and the development is extremely rapid. This paper will compare and analyze the cloud platform model.

\section{Comparative Research Framework}

Foreign scholars have done a lot of research on the connotation and composition of the space cloud platform. Pan Xinyu believes that the cloud platform is the abbreviation of the cloud service platform, which connects multiple cloud request terminals with cloud providers. Based on the cloud computing information platform architecture, Jia-bin Li divides the cloud service platform into infrastructure layer (IaaS), cloud business layer (PaaS), cloud application layer (SaaS) and user client. Radhika et al. believe that the cloud platform is an ecological network created and integrated by leading companies, attracting network members and potential members through big data tools. Kindstrom believes that the cloud platform model involves new business models, new 
target markets, new competitors, new value networks, new value chains, and new revenue channels. Zhang Yuming and others believe that the cloud platform is composed of the following main elements, including users, partners, third-party enterprises and other related organizations to form a cloud-based collaborative innovation platform, with the shortest development cycle and the most reasonable cost. Meet the individual needs of users. Ji Liuxiang and others believe that the maker space cloud platform is based on the cloud platform to provide platform components and middleware to realize cloud entrepreneurship and sharing. [3-8] Referring to the above documents, this paper believes that the maker space cloud platform is a cloud intermediary service platform that connects between the cloud entrepreneurial provider and the cloud entrepreneurial demand end. It can effectively promote cloud transactions of entrepreneurial resources and entrepreneurial capabilities; it is mainly based on cloud foundation. The cloud foundation, cloud function layer, cloud resource layer and cloud target layer are composed of four parts, which ultimately realizes the sustainable development of the cloud. This paper establishes a comparative research framework of cloud platform model of maker space as shown in Figure 1.

\section{Cloud sustainability}

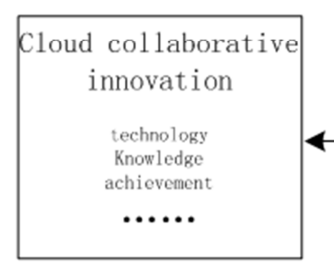

Target layer Cloud Coordination

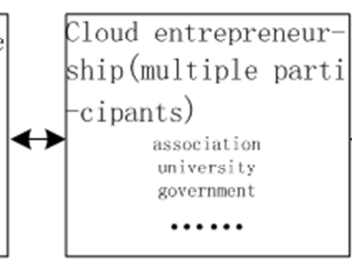

Resource layer Cloud shareing

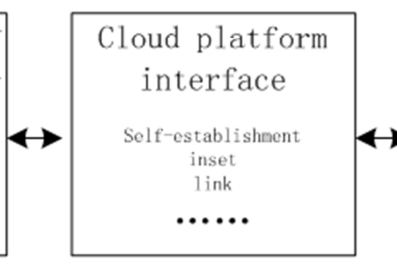

Functional layer Cloud business

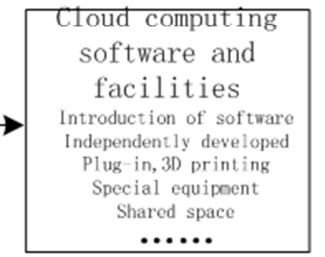

Base layer

Cloud computing etc.

Figure 1. The comparative research framework of cloud platform model of maker space.

In order to compare the maker space cloud entrepreneurship between China and foreign countries, this paper takes the characteristics of cloud entrepreneurship, data collection, and the development status of the region as the sample selection principle to form a comparative database. Since 2013, the US incubator professional rating agency, the Seed Accelerator Ranking Project, ranks TOP30 of the world's business incubators every year. This paper uses this as the basis for the selection of foreign samples, and then refers to the list of entrepreneurial incubators in other countries, and screens out a total of 48 incubators with the characteristics of entrepreneurial maker space cloud entrepreneurship as a list of foreign sample. The foreign sample banks are from the United States, the United Kingdom, Germany, Russia, Brazil, and other regions including Australia and South Africa. The sample database data is substituted into the SAS 9.4 software, and the output statistical descriptive results are organized as shown in Table 1 and Table 2.

Table 1. Statistical description of foreign maker space samples.

\begin{tabular}{|c|c|c|c|c|c|}
\hline variable & $\mathbf{N}$ & mean value & Standard deviation & Minimum value & Maximum value \\
\hline Cloud Venture Resources & 40 & 13.7 & 2.9 & 1 & 116 \\
\hline Cloud entrepreneurial function & 40 & 3.3 & 0.4 & 1 & 11 \\
\hline Cloud entrepreneurship collaboration & 40 & 1.8 & 0.4 & 0 & 10 \\
\hline $\begin{array}{l}\text { Cloud entrepreneurship sustainability } \\
\text { (revenue channel) }\end{array}$ & 40 & 2.1 & 0.1 & 2 & 4 \\
\hline
\end{tabular}

Table 2. Statistical results of domestic mass space samples.

\begin{tabular}{|c|c|c|c|c|c|}
\hline variable & $\mathbf{N}$ & mean value & Standard deviation & Minimum value & Maximum value \\
\hline Cloud Venture Resources & 40 & 25.6 & 44.5 & 3 & 310 \\
\hline Cloud entrepreneurial function & 40 & 8.0 & 7.6 & 2 & 41 \\
\hline Cloud entrepreneurship collaboration & 40 & 5.5 & 6.8 & 0 & 30 \\
\hline $\begin{array}{l}\text { Cloud entrepreneurship sustainability } \\
\text { (revenue channel) }\end{array}$ & 40 & 2.6 & 0.6 & 2 & 5 \\
\hline
\end{tabular}

\subsection{Cloud Base Layer}

The maker space cloud platform is based on cloud computing and the like, and is mainly composed of infrastructure and cloud computing software. Cloud computing infrastructure includes 3D printing facilities, dedicated test and development instruments and equipment, shared meeting and office space, and open community labs, workbenches, machine workshops, workshops or studios. Through the sharing of infrastructure, maker and entrepreneurs of different backgrounds can effectively use the conditions provided by maker space to create digital or 
creative products or services. Cloud computing software is currently built on the open source ecosystem. Developers use a variety of open source Devops (Development and Operations abbreviated, development and operation) tools to introduce or independently develop cloud computing software for the creation of space cloud platforms, and build automation. Infrastructure platforms (such as OpenStack and Kubernetes) to facilitate cloud application and delivery; for faster software development, developers are expanding on the space cloud platform to add new features and plug-ins to cloud services on AWS, Microsoft Azure and Migration between Google Cloud and more. [9]

\subsection{Cloud Function Layer}

Maker space platform provides various cloud business function interfaces. These cloud function interfaces are mainly divided into three categories: The first category is self-built cloud function, which is independently developed by maker space platform, and entrepreneurs can directly enter after clicking The core of the creation of the space itself; the second type is the embedded cloud function, the entrepreneur clicks on the functional interface and then links to the core cloud of other professional intermediaries. This type of embedded function is usually for professional needs, creating space. The third category is a simple web link, which is clicked by the entrepreneur to enter the partner's webpage, so that the entrepreneur can browse the new webpage and select the required functions and modules on the new webpage. The cloud function of maker space platform can best reflect the core competitiveness of maker space. Maker space usually uses cloud technology suitable for its own and sets cloud functions that reflect individual characteristics. For example, at the United Nations Headquarters Global Women's Summit, a cloud platform for female talent recruitment was introduced. It is an online platform formed by Transparent Business Cloud technology, matching algorithms and remote workforce management tools. Remote job opportunities are connected, leveraging on-demand cloud technology and data analytic capabilities to transparently hiring, monitoring, managing, collaborating, evaluating and paying transparently, enabling companies to find, hire and manage high-end women's talent through online platforms. [10]

\subsection{Cloud Resource Layer}

The cloud resource layer of maker space cloud platform is the basis for maker space to provide deep cloud services for entrepreneurs. According to the research in this paper, the following three types of cloud resources are mainly aggregated on the cloud platform: The first major type is to provide various types of entrepreneurial support resources online, including industry associations, university research institutions, government departments and other important support forces; The second major type is the online gathering of entrepreneurial tutor resources to provide professional guidance to entrepreneurs through online video or online Q\&A; The third type is online to provide a variety of entrepreneurial service resources, including business registration, financial taxation, competitive strategy, intellectual property, law, marketing, human resources recruitment, visual design, brand planning and other types of entrepreneurial service intermediary resources.

\subsection{Cloud Collaboration Layer}

Providing cloud collaborative innovation is an advantage that cloud platforms differ from traditional incubators. Entrepreneurs provide entrepreneurial needs through the cloud platform function interface. The cloud platform uses cloud computing technology to perform real-time search and intelligent matching in cloud sharing resources according to user needs, and provides on-demand services for entrepreneurs. Different maker space cloud platforms have different cloud technologies, different cloud functions, and different cloud resources. The cloud services provided to entrepreneurs are different and dynamic. In order to provide suitable and cheap resources, the cloud platform must effectively classify cloud resources and prepare for intelligent matching. [11-13] The cloud platform should be agile and flexible, and can effectively use big data analysis tools to identify and mine the different entrepreneurial needs of entrepreneurs at different startup stages. In this paper, the intelligent matching degree is taken as the measurement dimension of the collaborative performance of the cloud platform. The cloud demand side, the cloud platform cloud service and the cloud provider end achieve cloud collaboration through intelligent matching.

\subsection{Cloud Sustainability}

Cloud sustainability refers to the funds and benefits that support the sustainable development of the space through various income channels. According to the research in this paper, the revenue channels of maker space are divided into 12 categories: The first category is shared space rental fees, that is, rents are collected for shared office space; the second category is shared equipment usage fees, which are charged for the use of dedicated facilities; and the third category is general service fees, which means business services such as printing receive service fees; the fourth category is value-added service fees, including the provision of training, legal, accounting, consulting and other entrepreneurial value-added services to obtain returns; the fifth category is the mentoring fee, through one-on-one mentoring Instructor tutor fees, usually referred to as more complex activities, such as 3D design and printing, programming, etc; The sixth category is the income of exhibitions and fairs. The proceeds are obtained through booth fees and the sale of tickets. The seventh category is the equity income, that is, the original equity of the start-up enterprise is first invested, and then the proceeds are obtained by selling the appreciation of the equity; The eight categories are government subsidies, that is, subsidies are provided by governments or institutions at all levels; the ninth category is membership fees, including membership in various clubs and salons; and the tenth 
category is donation fees, which are funded through online donations from visitors. The eleventh category is the online sales income, which earns revenue from online sales of products produced by members; the twelfth category is other types of income-generating income.

\section{Comparison of Development Status}

Since the publication of the maker space in China in 2015, this paper began to collect and analyze the samples of Chinese maker space, randomly selected 610 samples of qualified samples for statistical analysis, and statistics on the development of Chinese maker space cloud platform model status quo. The US has created an early space development. From 2013, it began to randomly collect US space samples for 5 years and selected 610 samples for analysis to summarize the development status of the US space cloud platform model.

\subsection{Cloud Sustainability}

Chinese maker space in the cloud infrastructure, the shared use of space has become a universal model, $91 \%$ of the maker space can use shared meeting rooms, offices and so on. A small number of maker spaces provide intelligent services such as intelligent identification, cloud-controlled electricity meters, and cloud printing. Professional-oriented space creates a dedicated space, dedicated testing equipment, dedicated debugging equipment and intelligent monitoring. For example, Weichai truck power train maker space has innovative studios, technician studios, test centers, special performances. Laboratory, parts test bench, etc. In view of the cost of use and the technical threshold, Chinese maker space and partners are currently in the stage of gradual popularization, with free trials of domestic software, including uCloud, Alibaba Cloud, Tencent Cloud, Huawei Cloud and other public clouds. And EasyStack based on open source technology, Kyushu Cloud, HaiyunJiexun and Lingque Cloud based on Docker container technology. Cloud computing software involves artificial intelligence, hybrid cloud, live cloud, financial cloud, proprietary cloud, private cloud, mass computing, security, global services and other fields.

The US maker space is divided into five categories. Among them, the number of newly created makers in the past three years is the largest, according to the survey of more than 500. This kind of space creates and displays a wealth of specialized tools and equipment online, and members of the surrounding community can use it online or online. These specialized tools are more expensive equipment, including CNC equipment, including 3D printers, 3D scanners, CNC mills, laser cutting machines; metal processing equipment, including $\mathrm{CNC}$ milling machines, CNC plasma machines, drilling machines, welding Large equipment such as machines; advertising production equipment, including embossing machines, embossing slide machines, die-cutting machines, heat guns, special easels, etc.; electronic equipment, including Arduino development systems, sensors, oscilloscopes, electronic welding stations; woodworking tools, Including milling machines, wood lathes, woodworking drills, sanders, miter saws; glass fusion tools, including small furnaces and large furnaces; textile tools, including heavy-duty weaving machines, weaving machines, leather punching tables, Leather tools; ceramic tools, jewelry making equipment, food processing equipment, and electronic production platforms. These specialized tools are expensive and difficult for individuals to afford, but they are used in online sharing and online booking to create a reliable production site for all kinds of tangible creative products. In the US, such maker space is densely populated in major communities and serves the surrounding members. Some advanced special equipment has become standard equipment. For example, 3D printers are almost an indispensable tool for every maker space. They are equipped with one-on-one training on the design and use of instructors, and there are special types of $3 \mathrm{D}$ printers in the maker space. A wide range of dozens of creative designs can be printed in almost any kind, size, shape and performance. In the same way as China, the US has shared a variety of laboratory and office space, but $20 \%$ of these spaces also share kitchens and restaurants. The large-scale space creation in the US is based on the cloud computing software of the US giants, such as Google Cloud, AWS, Microsoft Azure, etc. Based on cloud computing software, some of the maker space also develops dedicated cloud computing software and cloud storage space. Plug-ins and function modules are embedded on the self-owned cloud platform to facilitate members to use cloud resources, cloud functions, and cloud migration and collaboration between various clouds.

\subsection{Comparison of the Status Quo of Cloud Function Development}

The cloud functions that China can provide involve more than 30 sub-categories, among which the top six cloud functions include online financing, online marketing planning, cloud technology, cloud accounting, and big data analysis. $31 \%$ of China maker space has opened online registration and online membership functions to provide exclusive services for online members. $15 \%$ used the online submission requirements feature, including online submission of business plans and 24-hour line performances, as well as the introduction of online social security and other cloud functions. Some of maker space also developed a dedicated app (mobile app) or set a public number for online and offline management. $80 \%$ of the chain-based crowd-creating space has launched online video, online meetings and online training. $51 \%$ of the creation space will embed or link the cloud function of the partner as a partner to the function interface of its own space to realize the transfer and docking of the demand. $48 \%$ of maker space can provide CRM (customer relationship management) system, order management system, enterprise mailbox system, regional operation online management system, commodity online management system, data analysis system, WeChat public number system, user management system, cloud warehouse online services such as management systems. 
The types of space cloud functions in the US are as high as 80 categories in China. The top six cloud functions are online financing, industry-university research, online training, association members, software development, and small business services. Compared with Chinese creative cloud space, the first type of cloud function is the same, but the last five different. The US attaches great importance to online training and software development. Each of the maker spaces has a series of training activities, software programming and development, and other salons, and members are invited to register online and participate on time. The function of the American Association of maker space members and the function of small business services are significantly different from those of China. Among the partners of the US Creative Space Cloud Function Providers, organizations that support small businesses or start-ups include: SBA Small Business Administration, SBN Small Business Network, SBDC Small Business Development Center, CAMEO Micro Enterprise Development Association, SCORE Small Business Guidance and Training and Small Business Research Centers (such as Missouri State University), State Government or Urban Small Business Offices (such as San Francisco), Small Business Economic Development Alliance, Lianbao Small and Medium Enterprises Association, Small Business Owners Health Insurance foundations, etc., can be seen that providing various types of association member services and special services for start-ups is an important cloud function of the US maker space. In addition, US maker space and start-ups are more inclined to cooperate with universities and scientific institutions to carry out original technology research and development, which is different from the tendency of China to cooperate with high-tech enterprises and develop practical technology research and development. The above differences illustrate the differences in the entrepreneurial environment between China and the US.

\subsection{Comparison of the Status Quo of Cloud Resources Development}

The cloud resources of Chinese maker space can be subdivided into more than 80 categories, among which the top 5 include fund companies, media, government organizations, high-tech companies, and law firms. According to Chinese cloud resources, Chinese maker space Cloud platform's most important cloud resources are online financing resources and the fund is the most favored financing channel. Secondly, it attaches importance to cooperation with the media. Chinese creative space is in a stage of rapid development, with special emphasis. The third is to pay attention to exchanges and cooperation with government organizations to win government policy support and cash support; the fourth is to pay attention to cooperation with high-tech enterprises, rather than cooperate with universities and scientific research institutions to illustrate Chinese maker space and Start-ups pay more attention to the development and cooperation of practical technologies. The fifth is to pay attention to cooperation with law firms, indicating that maker space and start-ups attach great importance to the use of legal firms to deal with contract disputes and prevent contract risks.

The top five cloud resources in the US are the online fund companies, associations, universities, government organizations, and online training companies. This shows that the US has created space for fund resources, association resources, university resources, government organization resources and online. Training company resources have a soft spot, which is basically consistent with the statistical results of the US space cloud function. The statistics of the online fund company's resource ownership rate of American maker space has an average of 6 in each creative space, while the average value of China is 3, indicating that the US fund companies are more developed, and also shows that US Resources are the core cloud resources. Another difference between American maker space and Chinese maker space in cloud resources is the internationalization of resources. Take the well-known American creative space YC as an example. More than half of its breeding projects come from other parts of the US, while international projects account for an average of nearly 20\%. However, Chinese maker space is significantly less on international resources. At the same time, the US created space cloud resources to focus on the vertical field. According to the statistics in this article, they subdivide cloud resources into more than 40 vertical areas, including artificial intelligence, short video, sharing economy, film and television, block chain, O2O, information technology, big data, Internet of Things, intelligent hardware, art design., social services, mobile internet, real estate hotels, TMT, cloud computing, software engineering, new energy, internet, agriculture, high-end manufacturing, environmental protection, catering services, big consumption, education, finance, automobile transportation, real estate services, medical health, tourism, local life, games, advertising marketing, hardware, cultural entertainment, corporate services, social networking, e-commerce, tool software, sports, logistics, etc. Each maker space only focuses on the accumulation of cloud resources in a certain vertical area, so that it's positioning and cloud resource features are more prominent and obvious.

\subsection{Comparison of the Status Quo of Cloud Collaborative Development}

Chinese maker space cloud collaboration includes collaborative collaboration, collaborative marketing, and collaborative lending. Collaborative office refers to office online editing, which provides fine management of permissions and versions, and can send large files to customers with one click. Collaborative marketing refers to the rapid release of demand through the intelligent marketing collaboration platform. The marketing experts and the media in the background complete the customized marketing plan for online demand, thus quickly connecting online and offline media, effectively reducing marketing costs. Collaborative loan refers to the online submission of loan name, loan amount, 
contact information, etc., and the professional team completes the evaluation, guarantee and docking online to help small and medium-sized enterprises to finance online.

American maker space pursues cloud collaboration in the vertical field. The team of instructors in the US is very large, such as the founder institute has more than 9,000 mentor and founder around the world. Faced with so many vertical areas, and such a large team of instructors, how do start-up filter cloud resources and mentors? To this end, an important technology currently adopted by the US maker space is intelligent matching technology, which is also an important internal development logic of the US maker space. They use artificial intelligence technology to find the most suitable matching object according to user needs. This technology has been widely used in the field of cloud collaboration. For example, American maker space ConceptDrop uses advanced matching technology to intelligently select outsourcers from cloud resources according to the outsourcing creative needs of different entrepreneurs, and find the best price, best deadline and quality for start-ups. For example, the company Sharpest Minds, which invested in YC in 2018, is based on the use of artificial intelligence to complete talent recruitment. The company has designed a series of programming tests, using artificial intelligence algorithms to find personality and professionalism according to the recruitment needs of start-ups. The most technically matched candidate.

\subsection{Comparison of the Status Quo of Cloud Sustainability}

Chinese maker space needs a stable income channel for cloud sustainability. According to the research in this paper, there are four main sources of income in Chinese maker space: rental income, government subsidies, service income, and investment income. Rent income is the income of space leasing, $80 \%$ of maker space relies on this channel as a source of income; government subsidies are cash support or policy concessions given by governments at all levels; entrepreneurial services include legal financial personnel, business registration, project positioning, service income, such as technical support, product promotion, media promotion, and road shows activities; investment income refers to the equity investment of maker space in the selected projects, and the proceeds are sold or transferred after the equity appreciation.

According to the research in this paper, in order to maintain cloud sustainability, US maker space has as many as 12 revenue channels. $90 \%$ of US maker space uses the following revenue channels: shared space charges, online rental special equipment, membership fees, training and other value-added service charges. US maker space advocates public welfare, never advertises how much money it has earned, but how many people have helped solve employment, how many companies have raised government support funds, how many children or young people have completed training, and how many companies have completed mergers and acquisitions. As the most important performance indicators for statistics and publicity, the enthusiasm of members to donate online is increasing. At present, more than $90 \%$ of US maker space adopts online donation methods, ranging from a few dollars to tens of thousands of dollars. And it can be used to offset taxes. They even mobilized members to donate specialized equipment online. In addition, the US to create space for the mentor one-on-one guidance are clearly marked with price tag, also through the exhibition, trade fairs, camping activities, etc. to collect service fees, through the sale of the investment equity of the possession of the start-up companies to obtain income, as well as for mergers and acquisitions, Data collection and other special activities charge service fees, forming a multi-channel revenue pattern.

\section{Difference Analysis Between China and America}

The cloud platform model has attracted great attention of Chinese work place. In recent years, it has been continuously explored and practiced, and formed its own characteristics. Stones form other hills may serve to polish the jade of this one, Compared with the cloud platform model of American maker place, China has the following differences in cloud foundation, cloud function, cloud resources, cloud collaboration and cloud sustainability.

\subsection{Compared with the US, Chinese Maker Space has Insufficient Cloud Professional Infrastructure}

In the past 3 years, the small-scale maker space that has been positioned as a community service in the US has sprung up, becoming an important gathering place for makers and entrepreneurs, and has played an important supporting role in promoting American public innovation and entrepreneurship. This kind of maker space is mainly characterized by the sharing of special facilities, and advanced equipment such as $3 \mathrm{D}$ printers has become popular and become a standard. The maker space is regarded as a place where people can pool their ideas to make innovative and creative products by sharing technology platforms, facilities, instruments and equipment. In China, $80 \%$ of the maker space takes renting and sharing office as the main source of revenue. It is revealed from the side that most of the crowd creation space in China is still at the level of shallow service such as renting, and the sharing professional facilities provided are insufficient. For example, 3D printers and various types of special production facilities and testing tools are difficult for individuals to undertake, they are indispensable infrastructure for the creation of the space cloud platform model. However, currently, such facilities or equipment cannot be generally provided by China maker space, which restricts the design and creation of new products.

\subsection{Compared with the US, the Cloud Function of Chinese Maker Space Cannot Meet the Explosive Growth Needs of Users}

American maker space pursues online interaction, emphasizing the role of various associations in the cloud platform and the cloud service functions for small and medium-sized enterprises. Chinese maker space should learn 
from this cloud service function. Under the new situation of "everyone is interconnected and everything is interconnected", there is a huge demand for the intelligent function of space creation, but this demand cannot be effectively met in China. The development of Chinese maker space cloud function is relatively lagging. At present, it mainly provides basic services, and some only provide online property leasing, while cloud functions such as intelligent management, online training, cloud accounting, cloud marketing, and device sharing are still lacking. The low satisfaction of start-ups in the maker space cloud functions is mainly reflected in two aspects: On the one hand, there are few work resources that can be shared; on the other hand, it is unable to provide online and offline services, insufficient activity carrying capacity, insufficient staff concentration and lack of activity. [14, 15]

\subsection{Compared with the US, the High-end Cloud Resources of Chinese Maker Space Are Still Scarce}

The American maker space cloud platform has rich international resources. They subdivide cloud resources into more than 40 vertical fields. Each maker space only chooses the vertical professional field that is good at it, and continuously integrates and accumulates vertical resources in the upstream and downstream. It has formed a distinct positioning and cloud resource characteristics, and it is easier to attract entrepreneurs in the field. Compared with the US, the rapid development of Chinese maker space benefits from the government's support policy effect, which brings a large number of competitors to enter, but the scarcity of high-quality cloud resources and other issues still need to be resolved. Mobile internet technology has penetrated into all fields of China, but the current dilemma is the lack of professional and shared resources such as industry associations. The problem faced by the new-born technology-based small and medium-sized enterprises in China is the shortage of shared intermediary service organizations, such as technical consultation, technical services, intellectual property consultation, productivity promotion centers and other intermediaries, as well as various funds and companies that can provide venture capital, micro-loans, technology guarantee and technology re-guarantee. The maker space in China has formed different clusters and layouts, some rely on the "University City" to have the advantages of resources such as talents and scientific research, but they are short-term in investment and financing resources; some are rich in investment and financing resources but lack intermediary and other service resources, so integrating cloud resources and sharing cloud resources is the focus of the coordinated development of the maker space cluster. [16-18]

\subsection{Compared with the US, the Cloud Collaboration Level of Maker Space in China Needs to Be Improved}

The pursuit of public space in the US is the intelligent matching under the premise of resource gathering. Compared with the US, the cloud collaboration level of maker space in China should not only stay on the surface, but also be value-oriented. The key point is to learn from the internal logic, realize the synergy of large data level, and apply the data of industry subjects to the analysis of large data of industry, use these real data to judge the direction of industrial upgrading, identify individual credit value, and solving the financing difficulty of new enterprises. Chinese maker space is still in the primary stage of the ecological system, mainly manifested as "emphasizing incubation, despising creativity". In the application modes of experience, experiment and inspection, there are problems such as insufficient participation and homogenization of multiple users. Its role in technological innovation and application innovation is relatively limited. Maker space can not only build a shared office space, but also become a hot land for venture capital and a space for nurturing innovation. The fundamental reason why maker space gathers Internet entrepreneurs is that it can provide "hidden knowledge" such as social relationship construction, innovation and skill learning. These "hidden knowledge" can only be formed into capital network and innovation network through extensive communication and transmission between entrepreneurs and investors. [19, 20]

\subsection{Compared with the US, the Cloud Sustainability of Chinese Maker Space Cloud Platform Model Is Constrained}

The US has formed 12 revenue-generating channels. While Chinese maker space currently faces a problem that is the profit model is single. The rental income is the main source of income for the maker space. In some of the research space, some rental income even exceeds $90 \%$ of total revenue, which will lead to the lack of ability and motivation to provide in-depth services. The sustainability of public space depends on the reasonable cost of cloud services and the high frequency of users, not the high cost or low frequency. At present, some crowd innovation spaces mainly rely on government subsidies to survive. The increase in the amount of government subsidies can only alleviate short-term demand and fail to meet the higher-level needs of start-ups. [21, 22]

In summary, the development of Chinese maker space has experienced a spurt development and entered the deep cultivation mode. Although Chinese maker space cloud platform has the advantages of latecomer, a variety of entrepreneurial entities, multiple types of innovative resources and multi-layer innovation networks have formed a preliminary cloud ecosystem, it is still confronted with the puzzles of cloud foundation, cloud resources, cloud functions, cloud collaboration, cloud sustainability and so on. The advanced practical experience and internal development logic of the US are worthy of our reference. [23, 24]

\section{Conclusion}

Comparing with China and the US, we can see that the number of maker space in both China and the US has increased rapidly. More than 30 types of cloud functions have been 
gathered, more than 80 types of cloud resources have been gathered, cloud collaboration network has been formed, and the cloud platform ecosphere of sustainable development has initially been formed. Chen Dejin believes that the development of space creation in China is still facing certain challenges. Due to the lack of effective rules, the top-level design is not perfect, the innovation is insufficient, and the characteristics are lacking. The foreign maker space has its own background, development model and industrial characteristics, especially in foreign countries. Under the influence of innovative culture and innovative spirit, the government's strong support has greatly promoted the growth of the space of maker. [1] Xie Xuefang and other scholars believe that China's maker space incubation function is limited, the incubation efficiency is low, and its services are mostly concentrated in the basic service stage, and some even play the role of "two landlords". And the equipment supply, training and counseling required for enterprise development, functional services such as financial assistance and marketing are not performing well. This is mainly because China's maker space is a government-led model. Its development model is mostly borrowed from the incubator. The incubator is mostly invested by the government. The source of funds depends on the technology innovation fund, and its own profit level is low. In addition, many newly established Maker Space regards the government dividends, tax reduction and other policy dividends as the driving force for creation, and the profit model has not yet been established. [14] The American maker space cloud platform model developed earlier and accumulated certain development experience, The Chinese maker space cloud platform model should learn from two aspects of "surface" and "interior".

\subsection{The Enlightenment of the External Form of American Maker Space Cloud Platform}

American maker space brings inspiration to China in terms of cloud infrastructure, cloud functions, cloud resources, cloud collaboration, and cloud sustainability: First, in terms of cloud infrastructure, the development trend of the US enlightens that China should vigorously develop community-based, small-scale and subdivided areas of professional mass-creation space, which should account for a large proportion in quantity and equipped with specialized facilities to provide convenient services for neighboring communities or members, and gradually developing into an innovative and entrepreneurial platform based on the segmentation of professional fields or surrounding communities, forming a fundamental innovation platform and network for innovation; Second, in terms of cloud function, to learn from the development experience of the US, China should improve the cloud technology and cloud resources for start-ups to interact with funds and mentors online, and support the development of various small and medium-sized enterprise development associations, so that small and medium-sized enterprises can get professional guidance and support through online interaction, to avoid detours in the process of entrepreneurship; Third, in terms of cloud resources, drawing on the internal mechanism of the American cloud entrepreneurial platform, in order to better provide vertical search service for start-ups and cloud resource docking service between upstream and downstream, Chinese maker space should be positioned in the professional field, integrate cloud resources in some vertical fields, and attach importance to the accumulation of international resources; Fourth, in terms of cloud collaboration, the experience of American maker space in pursuing intelligent matching suggests that Chinese maker space should pay attention to the development and application of intelligent matching software technology, speed up the accumulation of space resources in the cloud, and learn from the talent selection, fund selection, tutor selection, and outsourcing choices to learn from the intelligent matching technology of American maker space to improve the efficiency of innovation and entrepreneurship; Fifth, in terms of cloud sustainability, The 12 income generating channels in the US are also worth to reference. Compared with the revenue channels of American maker space, Chinese maker space has a larger space to expand in the following 7 channels: Online rental of special equipment, online donation, mentor one-on-one guidance, exhibitions, trade fairs and camping activities, equity investment and transfer, online sales of creative products, and other channels such as mergers and acquisitions, big data analysis, etc., to form a multi-channel revenue to protect the viability and sustainable development of Chinese maker space. Learning from the experience of the US, promote the sustainable development of all kinds of crowd innovation space through different levels and different channels, and implement hierarchical development support policies according to the scale and positioning of the crowd innovation space: for localized mass-creation space, it is advisable to focus on supporting the purchase of specialized equipment and serving the entrepreneurs and entrepreneurs of surrounding communities; for the maker space suitable for different localization, it is appropriate to support its large-scale activities, support its chain-like development, continuously improving its scale and influence, and ultimately moving towards internationalization.

\subsection{The Enlightenment of the Internal Development Logic of American Maker Space Cloud Platform}

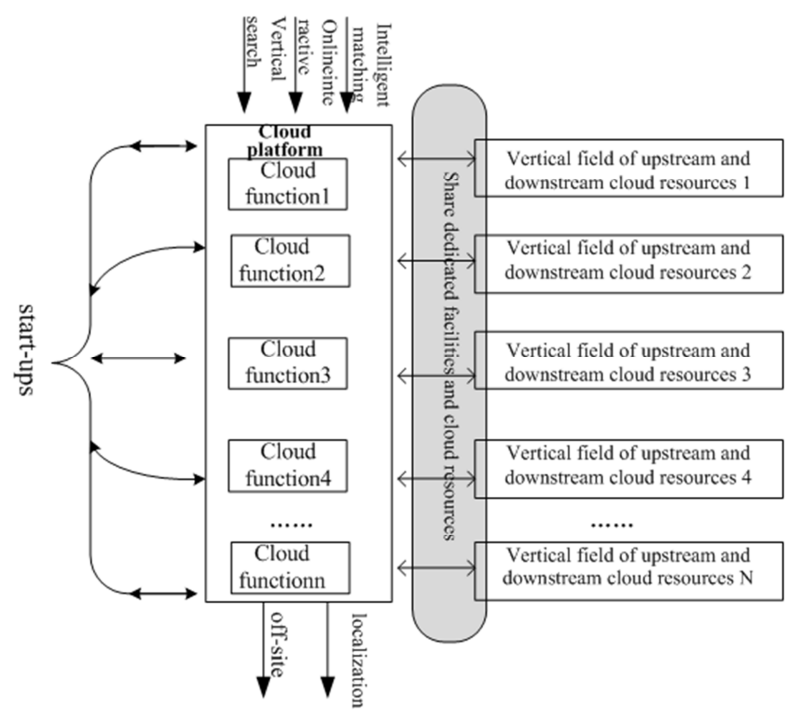

Figure 2. The internal logic of American maker space cloud platform model. 
The internal logic of American maker space cloud platform model is shown in Figure 2. Through the in-depth analysis of the development status of American maker space cloud platform, we can find that the internal logic of its development mainly shows five characteristics: professional facility sharing, vertical search, online interaction, intelligent matching and hierarchical development. First, professional facility sharing, American maker space has abundant specialized facilities, which are expensive and maximize their use value through shared use. Chinese maker space should learn from the practice of sharing professional facilities to change the scarcity of resources of special facilities and reduce the threshold of their use. Second, vertical search, American maker space cloud platform can be divided into more than 40 vertical domains, these domains are connected upstream and downstream, while American maker space is mostly located in a vertical domain. China should vigorously develop the space professionalism, focus on serving a vertical field, and start-ups can use the cloud function vertical search of the cloud platform interface to quickly find the cloud resources of interest in the vertical field. Third, online interaction, more than $90 \%$ of American maker space has opened membership functions, Chinese maker space should vigorously develop membership functions and support members to increase online training efficiency through online interaction with tutors and funds. Fourth, intelligent matching, American maker space cloud collaborative innovation is based on intelligent matching, such as talent recruitment, financing, outsourcing and mentoring, all of which are intelligently matched from cloud resources. Chinese maker space should pay attention to the development of intelligent matching technology, which is convenient for start-ups to find optimized cloud resources in a short period of time to save the time cost of start-ups. Fifth, hierarchical and sub-channel development, American maker space focuses on serving local community members and realizes sustainable development through revenue channels such as membership fee, facility rental fee and donation fee, with the goal of service localization development; while well-known joint office space is committed to chain and internationalization. Chinese maker space should subdivide the level, develop online applications, road shows and other forms to attract foreign resources, achieve remote chain replication, and then achieve profit through investment, transfer, mergers and acquisitions, form a cloud platform ecosystem, continuously promote alienated development, and ultimately achieve international expansion.

In a word, cloud platform model is the trend of future development of maker space. Drawing on the development experience of the American maker space cloud platform, Chinese maker space cloud platform model should learn from two aspects of "surface" and "interior", except the external forms of cloud foundation, cloud function, cloud resources, cloud collaboration and cloud sustainability, we should also learn from the internal development logic of professional facilities sharing, vertical search, online interaction, intelligent matching and hierarchical development, and will eventually provide a sustainable cloud platform ecosystem for public innovation and entrepreneurship in China.

\section{Acknowledgements}

This paper is the result of the Beijing Municipal Social Science Fund Key Project "Study on the Incentive, Conduction and Diffusion Mechanism of Beijing Maker Space Innovation Power Source". (17GLA007)

\section{References}

[1] Chen dejin. Comparative Analysis and Empirical Enlightenment of Foreign Maker Space Business Models [J]. Science Management Research, 2017, (03), 110-113. (in Chinese).

[2] Wang youmei, Ye Ailing. From maker space to mass creation space: functional model and service path based on innovation 2.0 [J]. Research of Audio-Visual Education, 2015, (11): 5-12. (in Chinese).

[3] Pan xinyu. Dynamic Strategy Analysis of Manufacturing Capacity Sharing in Cloud Manufacturing Environment [J]. Industrial Economy, 2016, (05): 16-29. (in Chinese).

[4] Jia-bin Li, Shi-wei He, Wei-chuan Yin. The study of pallet pooling information platform Based on Cloud Computing [J]. Scientific Programming. 2018, (05): 1-5.

[5] Radhika C, Kousalyadvi M, Balamurugan V. Business ecosystems - to dominate in the modern business [J]. IJSR -International Journal of Scientific Research, 2013, (05): 354-355.

[6] Kindstrom D. Towards a service--based business model: key aspects for future competitive advantage $[\mathrm{J}]$. European Management Journal, 2010, 28 (06): 479-490.

[7] Zhang yuming, Wang fuyao. The Construction Elements and Implementation Path of Cloud Innovation Model--Based on the Case Study of Huawei Company [J]. Science and Technology Progress and Policy, 2016, (01): 16-21. (in Chinese).

[8] Jiliuxiang, Sheng Ge. Theoretical Foundation and Planning Framework of Cloud Entrepreneurial Ecosphere [J]. Chinese Journal of Management, 2015, (11): 1646-1653. (in Chinese).

[9] Preimesberger, Chris. Cloud 2020: canonical's six trends shaping the future of cloud [N]. eWeek, 5/22/2018.

[10] PR Newswire. A global cloud--based platform to connect professional women with flexible remote job opportunities is unveiled at the United Nation Event in New York City today [N]. PR Newswire US, 03/15/2017.

[11] Tao F, Cheng Y, Xu L D, et al. CCIoT -CMfg: cloud computing and internet of things based cloud manufacturing service system [J]. IEEE Transactions on Industrial Informatics, 2014, 10 (02): 1435-1442.

[12] Tao F, Zhang L, Venkatesh V C, et al. Cloud manufacturing: A computing and service--oriented manufacturing model [J]. Journal of Engineering Manufacture, 2011, 225 (10): 1969 -1976.

[13] Xu X. From Cloud Computing to Cloud Manufacturing [J]. Robotics and Computer-integrated Manufacturing, 2012, 28 (01): 75-86. 
[14] Xiexuefang, Liu qing-liang. Ecological model of maker space in the innovation 2.0 era - comparison and enlightenment of China and foreign countries. Science research. 2018, (04): 577-585. (in Chinese).

[15] Zhao yijing, Qianqinglan. Current characteristics and satisfaction evaluation of maker space in guangzhou $[\mathrm{J}]$. Science and technology management research, 2017, (22): 93-98. (in Chinese).

[16] Ouguoli, Wang ruizhe. Difficulties and countermeasures faced by the rapid development of Chinese sharing economy [J]. Economic aspect, 2017, (05): 54-59. (in Chinese).

[17] Shao chuanlin, Wang liping. Research on the development path of science and technology finance from the perspective of innovation-driven [J]. Economic aspect, 2016, (11): 65-69. (in Chinese).

[18] Zhang danning, Fu xiaoyun, Yi pingtao. Research on the development path of maker space industrial cluster in shenyang city -- based on operational efficiency measurement. Journal of northeast university (social science edition). 2017, (1): 34-40. (in Chinese).

[19] Xiao yan, Mengjian. Research on "intelligent upgrading" of Chinese cultural industrial parks from the perspective of big data [J]. Economic aspect, 2017, (09): 112-116. (in Chinese).
[20] Wang bo, Zhen feng, Zhu xianqiang. Connotation, development and planning strategy of Internet maker space -based on investigation and analysis of Shanghai $[\mathrm{J}]$. Urban planning, 2017, (09): 30-37. (in Chinese).

[21] Zhengjuan, Konggangcheng. Current situation and characteristics of maker space with regional industrial characteristics - a survey on crowd innovation space and young entrepreneurs in chaoyang district, Beijing [J]. Journal of Beijing union university (humanities and social sciences edition), 2018, (04): 61-67. (in Chinese).

[22] Chen wu, Li yanping. Cultivation of platform organizational competitiveness from the perspective of embeddability -- a multi-case study based on maker space [J]. Journal of economic management, 2018, (03): 74-91. (in Chinese).

[23] Li zibiao, Liu shuang, Liu leilei. The value-added path of maker space to cultivate incubated small and medium-sized enterprises - experience from 69 crowd innovation Spaces in tianjin $[\mathrm{J}]$. Science and technology progress and countermeasures, 2018, (03): 73-79. (in Chinese).

[24] Lvbo. Research on effective stimulation variables of virtual business incubator - based on CAS model and statistical comparison data $[\mathrm{J}]$. Science and technology progress and countermeasures, 2018, (02): 17-25. (in Chinese). 